\title{
Ginkgo biloba's footprint of dynamic Pleistocene history dates back only 390,000 years ago
}

\author{
Nora Hohmann ${ }^{1,2}$, Eva M. Wolf ${ }^{1}$, Philippe Rigault ${ }^{1,4}$, Wenbin Zhou ${ }^{3}$, Markus Kiefer ${ }^{1}$, Yunpeng Zhao ${ }^{3^{*}}$,
} Cheng-Xin $\mathrm{Fu}^{3}$ and Marcus A. Koch ${ }^{1 *}$ (D)

\begin{abstract}
Background: At the end of the Pliocene and the beginning of Pleistocene glaciation and deglaciation cycles Ginkgo biloba went extinct all over the world, and only few populations remained in China in relict areas serving as sanctuary for Tertiary relict trees. Yet the status of these regions as refuge areas with naturally existing populations has been proven not earlier than one decade ago. Herein we elaborated the hypothesis that during the Pleistocene cooling periods G. biloba expanded its distribution range in China repeatedly. Whole plastid genomes were sequenced, assembled and annotated, and sequence data was analyzed in a phylogenetic framework of the entire gymnosperms to establish a robust spatio-temporal framework for gymnosperms and in particular for G. biloba Pleistocene evolutionary history.

Results: Using a phylogenetic approach, we identified that Ginkgoatae stem group age is about 325 million years, whereas crown group radiation of extant Ginkgo started not earlier than 390,000 years ago. During repeated warming phases, Gingko populations were separated and isolated by contraction of distribution range and retreated into mountainous regions serving as refuge for warm-temperate deciduous forests. Diversification and phylogenetic splits correlate with the onset of cooling phases when Ginkgo expanded its distribution range and gene pools merged.

Conclusions: Analysis of whole plastid genome sequence data representing the entire spatio-temporal genetic variation of wild extant Ginkgo populations revealed the deepest temporal footprint dating back to approximately 390,000 years ago. Present-day directional West-East admixture of genetic diversity is shown to be the result of pronounced effects of the last cooling period. Our evolutionary framework will serve as a conceptual roadmap for forthcoming genomic sequence data, which can then provide deep insights into the demographic history of Ginkgo.
\end{abstract}

Keywords: Evolutionary history, Phylogenomics, Ginkgo biloba, Pleistocene

\section{Background}

During the early Permian, approximately 300 million years ago (mya), Ginkgoatae started to evolve into more than 16 different genera. The genus Ginkgo first appeared in the middle Jurassic approximately 170 mya $[1,2]$. As indicated by fossil evidence, a worldwide northern hemispheric

\footnotetext{
* Correspondence: ypzhao@zju.edu.cn; marcus.koch@cos.uni-heidelberg.de ${ }^{3}$ The Key Laboratory of Conservation Biology for Endangered Wildlife of the Ministry of Education, College of Life Sciences, Zhejiang University, Hangzhou 310058, China

${ }^{1}$ Center for Organismal Studies (COS) Heidelberg/Botanic Garden and Herbarium Heidelberg (HEID), University of Heidelberg, Im Neuenheimer Feld 345, D-69120 Heidelberg, Germany

Full list of author information is available at the end of the article
}

radiation in temperate forests occurred during the Late Mesozoic and early Tertiary period about 65 mya [1]. However, at the end of the Pliocene and the beginning of Pleistocene glaciation and deglaciation cycles Ginkgo went extinct all over the world [1], and only few relict population areas remained in China serving as sanctuary for Tertiary relict trees [3].

There is excellent fossil record of Ginkgoatae (see $[3,4]$ for further references), and therefore it is not surprising that this gymnosperm tree is among the enigmatic "living fossils" fascinating humans for hundreds of years [5]. Ginkgo is a long-lived dioecious tree, and the oldest individuals known in China are estimated to be approximately

(c) The Author(s). 2018 Open Access This article is distributed under the terms of the Creative Commons Attribution 4.0 International License (http://creativecommons.org/licenses/by/4.0/), which permits unrestricted use, distribution, and 
1000 to 3000 years old [6]. These old Ginkgo trees are often close to human settlements and this also is an indication that the tree has always been playing an important role in medicine, food, ornamentation but also in culture and religion (e.g. [7, 8]). Since a first draft genome of Ginkgo biloba was published recently [9] more detailed evolutionary and functional studies may be conducted in the near future.

However, some key questions have not been answered yet such as the temporal dynamics of Ginkgo evolutionary history setting a baseline for any further future evolutionary analysis. The status of two major geographical regions in China as refuge areas with naturally existing populations has been proven only one decade ago [3], and it is exciting to study now a "living fossil" in its natural environment with other "tertiary relics", the warm temperate deciduous forests (WTDF). There is also a continuously increasing interest in the evolutionary history of woodland types of East Asia serving as sanctuaries for many relic tree species from various genera such as Cercidiphyllum, Davidia, Euptelea, Ginkgo, Metasequoia, or Tetracentron [10]. The woodlands that are of particular interest and are often considered tertiary relict vegetation and species assemblages, are referred to as (i) warm temperate deciduous (to evergreen) forests, and (ii) subtropical broadleaved evergreen forests. In particular, the evolutionary and Quaternary history of temperate deciduous species in subtropical China remains under debate. While it is accepted that there are several species from deciduous forest that survived in long-term refugial isolation in subtropical China [3, 10-12], it remains controversial whether these relictual populations underwent glacial admixture [13], or whether constituent species populations of these often montane forest habitats remained isolated over (at least) the latest glacial and interglacial periods $[14,15])$. Temperate deciduous forests have been shown to possibly have a wider distribution during the last glacial maximum (LGM, 20,000 years ago) compared to the present-day distribution of potential vegetation using modeling approaches, whilst warm-temperate ever-green forests had a more restricted and southern distribution, respectively [14], also indicating (i) long-term refugial isolation of (warm-) temperate evergreen taxa in subtropical China and (ii) 'cryptic' glacial survival of (cool-)temperate deciduous forest trees in North China.

In our previous contribution analyzing range dynamics between co-occurring Asian temperate trees from temperate deciduous forests [16], we elaborated on the relevance of three competing hypotheses that may explain the variation in the degree of expansion to former range limits in eastern Asia. The first hypothesis, introduced by Qian \& Ricklefs [13], postulates that during glacial periods populations merged and admixed at lower elevations (isolation with admixture). A second and alternative hypothesis argued that these populations remained isolated during glacial as well as interglacial periods (continual isolation) [14]. A third hypothesis is mediating between both [16] and postulates that life history traits can impose restrictions on the range dynamics and population genetic structure of temperate plants (e. g. [17]). Further contentious topics being discussed under these three hypotheses have been summarized by Zhao and colleagues [18]. The various hypotheses were tested for G. biloba, and key traits limiting range expansion were hypothesized such as a slow and complex sexual reproductive cycle, large seeds with slowly developing embryos, and largely extinct fruit/seed dispersers. In the same study, the latest split time of Ginkgo refuge areas was calculated to approximately 50 thousand years ago and with strong evidence for genetic connectivity prior to the split and asymmetrical gene flow between regions afterwards. However, large 95\% HPD (highest posterior density) ranged from 17 to 95 kya, and, therefore, the split could also be placed either in warming periods during the Last Interglacial (LIG, 120140 kya) or postglacial warming or in the last cooling phase. It appears that west-east directed regional Ginkgo populations have not remained genetically isolated during the entire last glaciation [16]. Effective population sizes of the western and eastern refuge areas were small with 416 and 802 individuals, respectively, and may be dated back to the LIG, whereas ancestral effective population size was estimated with 27,225 individuals [16]. Although plastid DNA sequence data based on a limited number of genes indicated deeper divergence patterns than 50 kya $[3,16]$, no divergence times have been provided so far. Demographic analyses of population genetic structure from nuclear DNA markers (AFLPs; $[3,5]$ and independently confirmed structure of gene pools using microsatellites [16]) failed to detect deeper spatial demographic patterns.

There are few studies from species from deciduous forests also showing signatures of expansion during cooling phases [10, 19], and taxa from temperate evergreen forests also exhibit patterns of range expansion [20] and show some similarities with G. biloba such as a deep east-west differentiation [16]. On the other hand, for few taxa from EBLF a reverse pattern has been reported with shrinkage of distribution during cooling phases such as during the LGM $[21,22]$ and postglacial range expansion [23].

To further enlighten these processes, we aimed to unravel a high-resolution temporal maternal evolutionary history of Ginkgo biloba considering our previous knowledge on distribution, refuge areas and demography. Ginkgo is not only a living fossil, but the entire respective lineage of Ginkgoatae has been isolated for more 
than 300 million years with G. biloba being the only remaining living representative [24, 25]. Consequently, phylogenetic and temporal reconstructions within the lineage are challenging because of missing appropriate outgroups.

The main goals of this study were: (1) To reconstruct a reliable phylogenetic tree to define phylogenetic positions among clades found within G. biloba. Although there are numerous fossils of Ginkgo and its relatives, these fossils are of no use for calibrating a molecular clock, because fossils are missing that can be assigned to the different present-day gene pools and lineages of extant Ginkgo biloba. Therefore, we intended (2) to estimate split times for internal nodes among Ginkgo genotypes (e.g. crown group age of G. biloba) using primary fossil constraints within a gymnosperm-wide phylogenetic analysis. The results were used for secondary calibration of a larger G. biloba data set to (3) elaborate on the hypothesis that G. biloba expanded repeatedly during cooling periods throughout the late Pleistocene, and that warming periods such as the LIG forced Ginkgo into refuge areas. In order to generate sufficient DNA sequence information from coding and non-coding regions we sequenced entire plastid genomes using a genome skimming approach for subsequent assembly and annotation of plastomes.

\section{Results}

\section{Sequencing and assembly of plastid genomes}

With our genome skimming approach we were able to recover almost complete plastid genomes for all 71 samples. Coverage ranged from $9 \times$ to $845 \times$ with an average of $102 \times$, and was generally higher for those samples with more raw data available (Additional file 1). Likewise, the number of missing base pairs was higher for samples with low coverage; most samples with coverage $<50 \times$ had uncovered bases, while few of those with coverage $>50 \times$ had missing data. The highest number of uncovered bases was 402, but only four samples had more than $50 \mathrm{bp}$ missing. Overall, 24 genes were affected by missing data and filtering for low coverage, however in most cases only few base pairs were excluded for a single sample. Libraries for two samples (DHAL6 and WCQF5) were prepared using a different kit and sequenced separately, however this did not affect the quality of sequencing.

\section{Network reconstruction reveals eight genetic clusters among extant Ginkgo}

The complete plastome alignment of 71 Ginkgo accessions comprised 138,921 base pairs (bp) excluding the second copy of the Inverted Repeat (IR) region and indels. A total of 135 polymorphic sites were identified, of which 15 were parsimony uninformative. Consistency index (CI) and retention index (RI) as calculated by PAUP [26] were $95.1 \%$ and $99.4 \%$, respectively (CI 93.7\% and RI 99.1\% calculated without autapomorphies). Network reconstruction revealed 17 individual haplotypes clustered into eight groups (A-H) (Fig. 1a), five of which contained more than one haplotype. Haplotype groups E-H were all closely related with only a few mutational steps separating the respective haplotypes. Haplotype groups A and B were also separated from each other by only a few substitutions, but deep splits were detected between those pairs as well as between groups $C$ and $D$. It is noteworthy that haplotypes of group $\mathrm{C}$ and $\mathrm{D}$ were only recovered from the easternmost population in the Tianmu mountains (TM), while haplotypes of group E$\mathrm{H}$ were completely absent in this population (Fig. 1b). These results are in congruence with our earlier studies $[3,16]$ showing a deep split between Eastern (Tianmu mountains) populations and the populations in Central and Western China (represented by the dashed lines in Fig. 1).

\section{Gymnosperm-wide phylogenetic reconstruction and divergence time estimation confirmed the cycad-Ginkgo sister relationship and revealed Ginkgoatae stem group age of approximately 325 mya}

To obtain secondary calibration points and topology information for our 71 Ginkgo accessions, we performed phylogenetic reconstruction and divergence time estimation using a subset of our Ginkgo samples in a gymnosperm-wide context based on the coding sequence of 78 genes $(60,520 \mathrm{bp})$, including protein coding genes and rRNA genes. Six Ginkgo accessions were selected to represent haplotype groups A-E and G. As haplotype groups E-H were closely related, only two representatives were chosen. Over the complete alignment, 17 sites from coding regions were polymorphic within Ginkgo, 6 of which were autapomorphies. In total, the gymnosperm-wide alignment comprised 28,905 polymorphic sites, of which 5355 were parsimony uninformative (CI 85.9\%, RI 85.1\%, excluding autapomorphies $84.7 \%$ and $84.0 \%$, respectively). Maximum likelihood (ML) phylogenetic reconstruction using RAxML [27] placed cycads at the base of gymnosperms, and Ginkgo as the sister to the clade of (Pinaceae(Gnetophytes,Cupressophytes) (Fig. 2). However, the bootstrap support was low and not significant for the most basal splits between Ginkgo and Pinaceae, while it was high for most nodes. The possibility of other topologies can therefore not be excluded.

The tree topology resulting from BEAST [28] analysis without constraints on tree topology suggested a sister relationship between Ginkgo and cycads at the base of the gymnosperms (Fig. 3). This relationship was also supported using the tree topology derived from the 


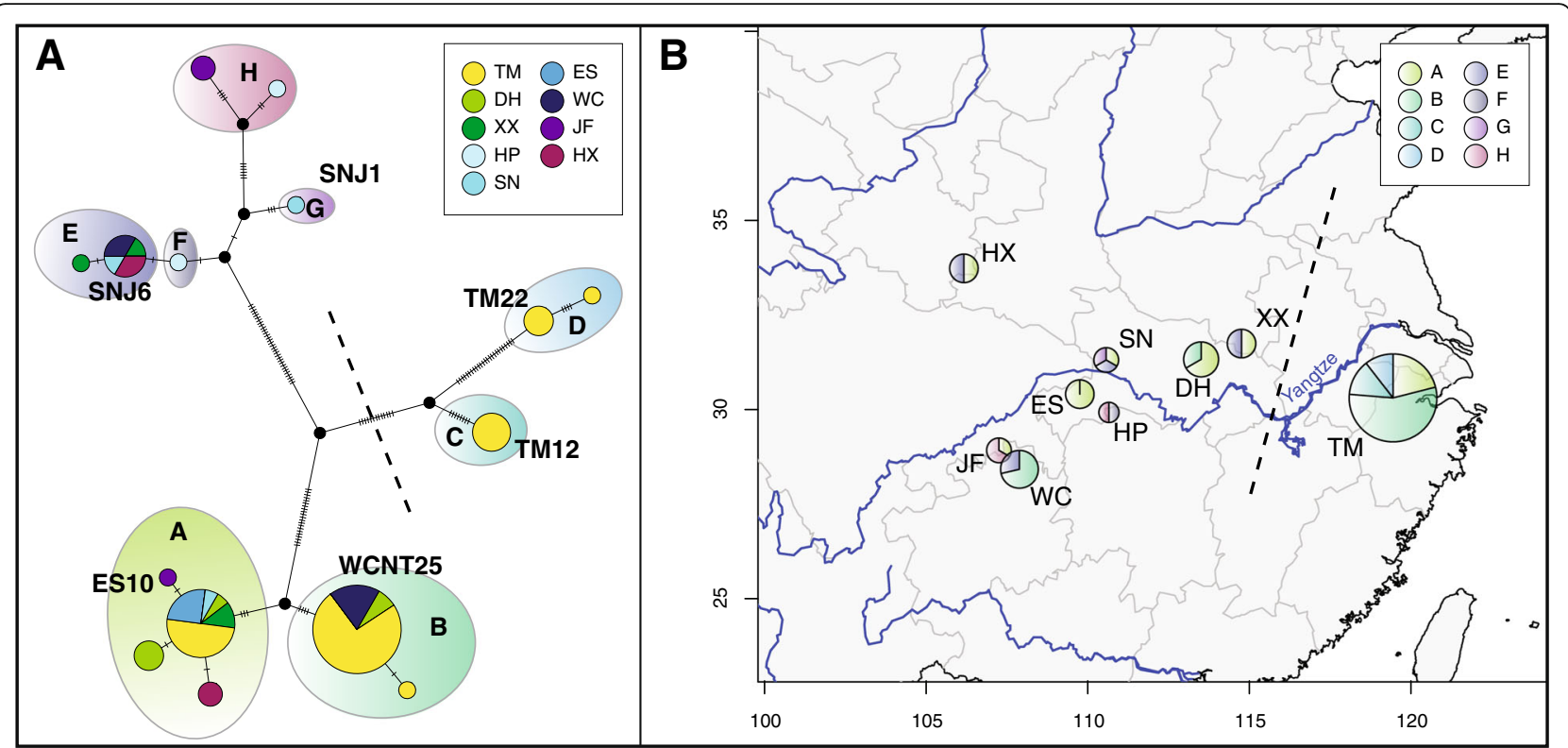

Fig. 1 Phylogenetic network reconstruction of 71 Ginkgo accessions. a TCS network based on complete plastomes. 17 haplotypes were clustered into eight haplotype group $(\mathrm{A}-\mathrm{H})$, different colors represent populations of origin. Six sample names denote the samples used in the gymnosperm-wide dataset. Size of haplotype pie charts is proportional to the number of individual sequences assigned to the respective haplotype. $\mathbf{b}$ Geographic distribution of the eight haplotype groups. Map was created with packages 'maps' [62], 'mapdata' [63] and 'mapplots' [64] in R version 3.2.3 [65] using the 'worldHires' map. Detailed geographic coordinates are provided with Additional file 1

RAxML analysis as starting tree for the BEAST analysis. Divergence between Ginkgo and cycads was estimated to 325 mya, and the crown age of Ginkgo was 0.52 mya using the speciation birth-death model.

Constraining the tree topology following the results from ML reconstruction or use of the yule tree model only had a very minor impact on divergence time estimates over all, particularly at younger nodes, and 95\% HPD intervals largely overlapped between analyses (Additional file 2). Within the Ginkgo clade, the distribution of age estimates overlapped between all four analyses, with the combination of birth-death model and constrained topology being slightly the youngest (Fig. 4a). We used the results from the birth-death unconstrained analysis (Fig. 4a) for secondary calibration at three nodes (Fig. 4b) and selection of the root position for the Ginkgo-only analyses. The birth-death model also performed better compared to the yule model in cycads plastome phylogenomics [29].

\section{Ginkgo phylogenetic reconstruction and divergence time estimation revealed successive divergence synchronized with Pleistocene cooling phases}

Maximum likelihood tree reconstruction of all 71 Ginkgo accessions was consistent with the network analysis and recovered all 17 haplotypes (Fig. 5). Bootstrap support was generally high for both deeper nodes and each of the eight haplotype groups $\mathrm{A}-\mathrm{H}$. The position of the root, which was set by selecting the clade comprising haplotype groups $\mathrm{E}-\mathrm{H}$ as the outgroup following the results from the gymnosperm-wide analysis, was also consistent with midpoint rooting and results from network analysis.

Divergence time estimation using the age distributions from the birth-death unconstrained gymnosperm-wide analysis as secondary calibration estimated the crown age of Ginkgo to 0.39 mya (95\% HPD: 0.22-0.79) (Fig. 6). The subsequent split occurred at 0.31 mya between haplotype groups A-B and C-D. The separation between haplotype groups $\mathrm{C}$ and $\mathrm{D}$ specific to Tianmu Mountain was much older than that between groups A and B (0.16 vs. 0.05 mya). The initial split within haplotype groups $\mathrm{E}-\mathrm{H}$ restricted to West China was also dated at 0.06 mya.

Comparing the occurrence of the various phylogenetic splits with the oscillation of glaciation (L1 to L4) and inter-glaciation/post-glaciation (S1 to S4), we found that all phylogenetic splits were placed into glaciation phases. In other words, any increase in net diversification is linked with cooling phases (Fig. 7).

\section{Low genetic variation of 35S rDNA loci within and among Ginkgo accessions}

The alignment of the rDNA sequences showed a very high degree of conservation, as expected. The overall alignment length was 6355 bp including 40 variable sites in total $(0.63 \%)$ distributed on the different building blocks of the 35S-rDNA locus (Table 1). 36 of these sites 


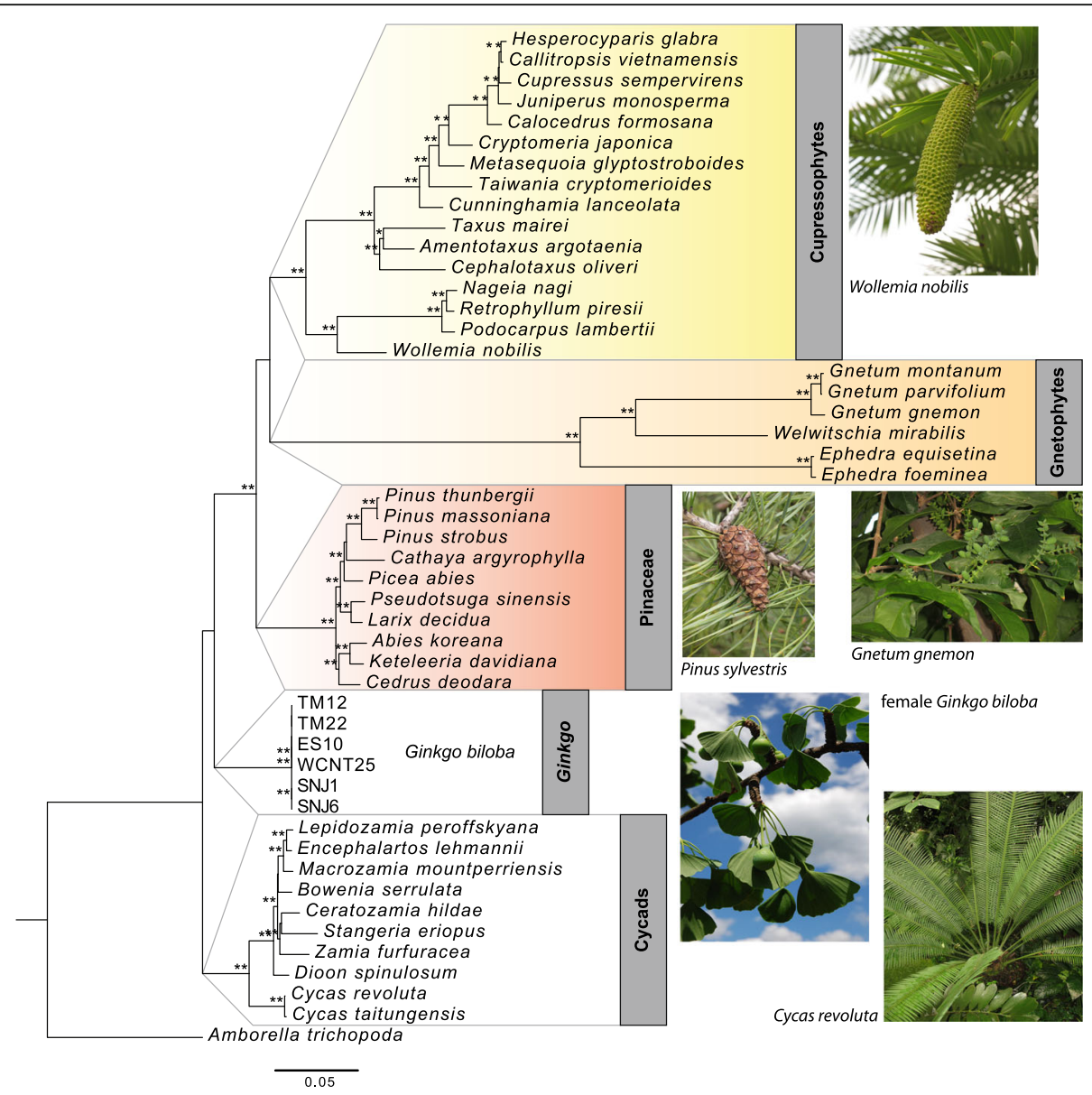

Fig. 2 Maximum likelihood phylogenetic reconstruction of Ginkgo in a gymnosperm-wide contextBootstrap values from 1000 bootstrap replicates are indicated $*^{* *}$ for bootstrap support of $100 \%$ and * for support of $\left.95-99 \%\right)$. Bootstrap support was $90 \%$ for the split between Ginkgo and the group of Pinaceae, Gnetophytes and Cupressophytes. Amborella trichopoda (angiosperms) was set as outgroup. Images taken by M.A. Koch

are variable within a single individual and represent intra-genomic variation of this high-copy number region.

Comparing among accessions there are 34 haplotypes with an overall total nucleotide diversity $(\pi)$ of 0.00048 . Analysis of read data for single individuals revealed that on average intra-genomic nucleotide diversity $(\pi)$ was 0.00074 with a standard deviation of 0.00024 . The alignment with all variable sites is provided with Additional file 3.

\section{Discussion}

Phylogenetic analysis of gymnosperms and the timeline for Ginkgo evolution

Phylogenetic relationships among the five extant seed plant lineages are still not fully resolved and remain uncertain. This includes a hypothesized sister-relationship of cycads to extant angiosperms [30,31] rather than the more widely accepted monophyly of gymnosperms (e.g. the most recent comprehensive survey: [32]). Within gymnosperms alternative phylogenetic scenarios have been proposed focusing either on the placement of gnetophytes or on the placement of Ginkgo compared to remaining gymnosperms. For gnetophyte placement this evolutionary lineage has been considered (i) as the sister to cupressophytes ("gnecup" hypothesis; [25, 33, 34]), (ii) as sister to Pinaceae ("gnepine" hypothesis; [32, 35-37]), or (iii) as the sister to the conifer clade comprising cupressophytes and Pinaceae ("gnetifer" hypothesis, [38]). With respect to the phylogenetic position of Ginkgo there is either a basal sister relationship with cycads assumed, setting those two lineages apart from the rest of the gymnosperms ("cycad plus Ginkgo" hypothesis; [33, 34, 39, 40]), or a paraphyletic placement among gymnosperms with cycads splitting off first, followed by Ginkgo and then followed by the remaining gymnosperms ("Ginkgo alone" hypothesis; [24, 25]. From the various phylogenomic analyses it has become evident that Ginkgo and cycads are most likely sister lineages and the majority of the most recent studies confirmed this hypothesis. Although our ML analysis was not able to significantly resolve this relationship, BEAST also recognized the cycads-Ginkgo sister-relationship when we 


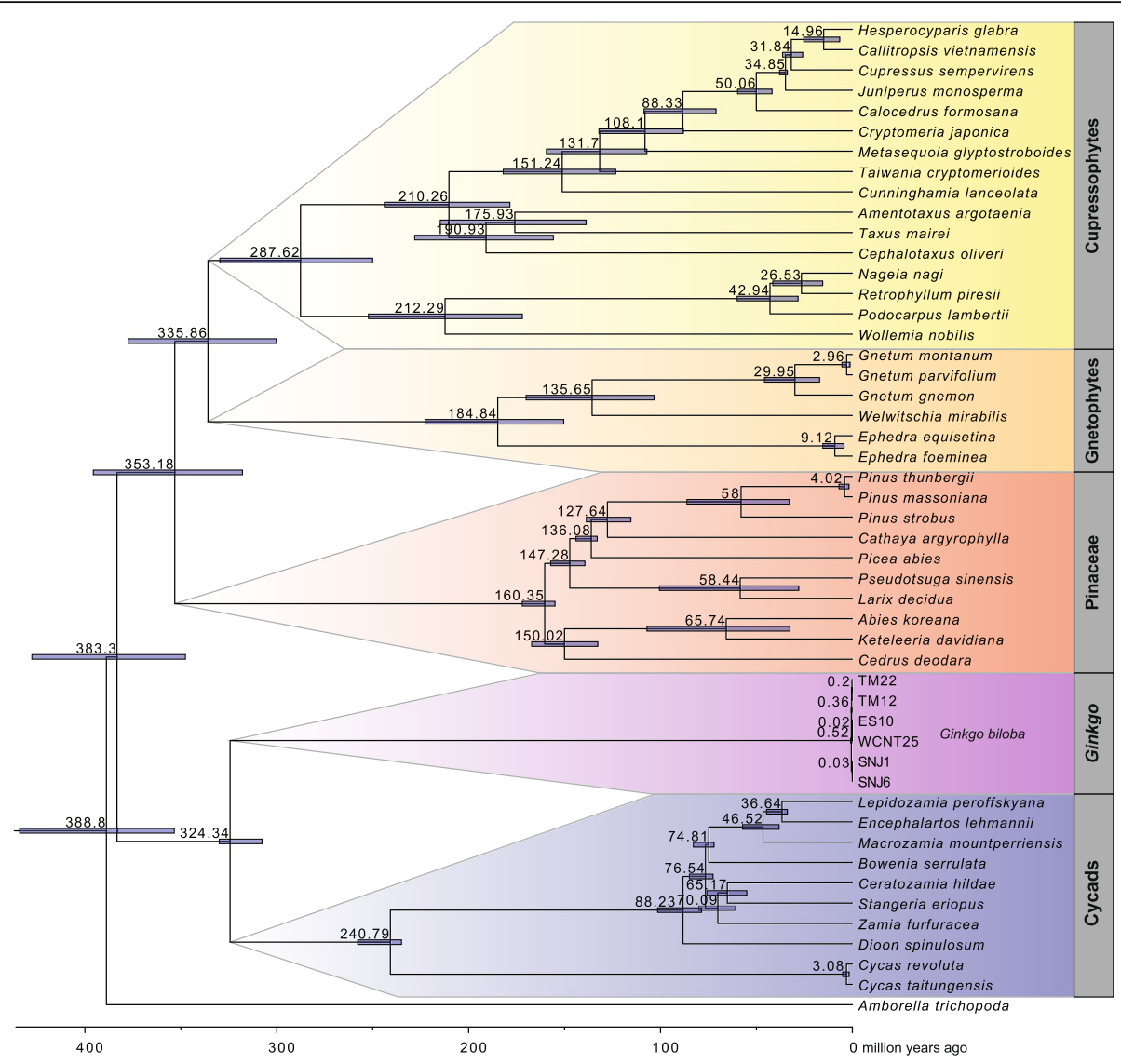

Fig. 3 Divergence time estimation of Ginkgo in a gymnosperm-wide context. Combined BEAST results from four independent MCMC runs of $5^{*} 10^{8}$ generations each. Calibration was based on 11 fossils (see Additional files 7 and 8). Divergence time estimates are shown with their $95 \%$ HPD intervals

used a starting tree based on the ML results for the Bayesian (BEAST) analysis. For phylogenetic relationships within gymnosperms "gnecup" and "gnepine" hypotheses are competing with each other, and it seems that the outcome not only varies among studies using the same source of DNA sequence information (e.g. either plastid or nuclear genomes), but also results from the different genomes may remain conflicting. In summary, it seems that plastid data tend to support the "gnecup" hypothesis [25, 33, 34], whereas data from the nuclear genome favor a "gnepine" topology [32, 33]. Our results are also in agreement with these findings. Both ML and BEAST trees based on our plastid genomes favored the "gnecup" hypothesis. The nuclear genome perspective remains unclear. The most recent results from the $1 \mathrm{KP}$ project [41] propose the "gnetifer" hypothesis for the nuclear genome perspective, which is in agreement with an earlier transcriptome based analysis focusing on early genome duplications in conifers and other seed plants [42]. However, $1 \mathrm{KP}$ data also support the "gnecup" topology for the plastid genome, which is again in agreement with the analysis presented herein. Based on our results we cannot contribute further to these discussions, but having reliably set our results into the framework of most up-to-date findings. This is important for our subsequently performed divergence time estimates and respective careful analyses.

Phylogenetic placement of Ginkgo is still under investigation with converging results as discussed above, and similarly the process of the temporal onset of the Ginkgoatae evolutionary lineage is under investigation with converging results. An extensive survey of fossils and justifications for using respective minimum and maximum constraints is provided in [24]. Using minimum/ maximum constraints set as 107.7 and 366.8 mya, respectively, they calculated the stem group age of Ginkgoatae with approximately 300 mya. This estimate was confirmed calculating a stem group age of Ginkgoatae with 304.5 mya [43]. For our study, it is important that we were able to confirm consistently this estimate with approximately 324 mya (Fig. 3), and, therefore further divergence time estimates within and among Ginkgo biloba clades are feasible. Also tree height (seed plants split/stem group age between present-day angiosperms 


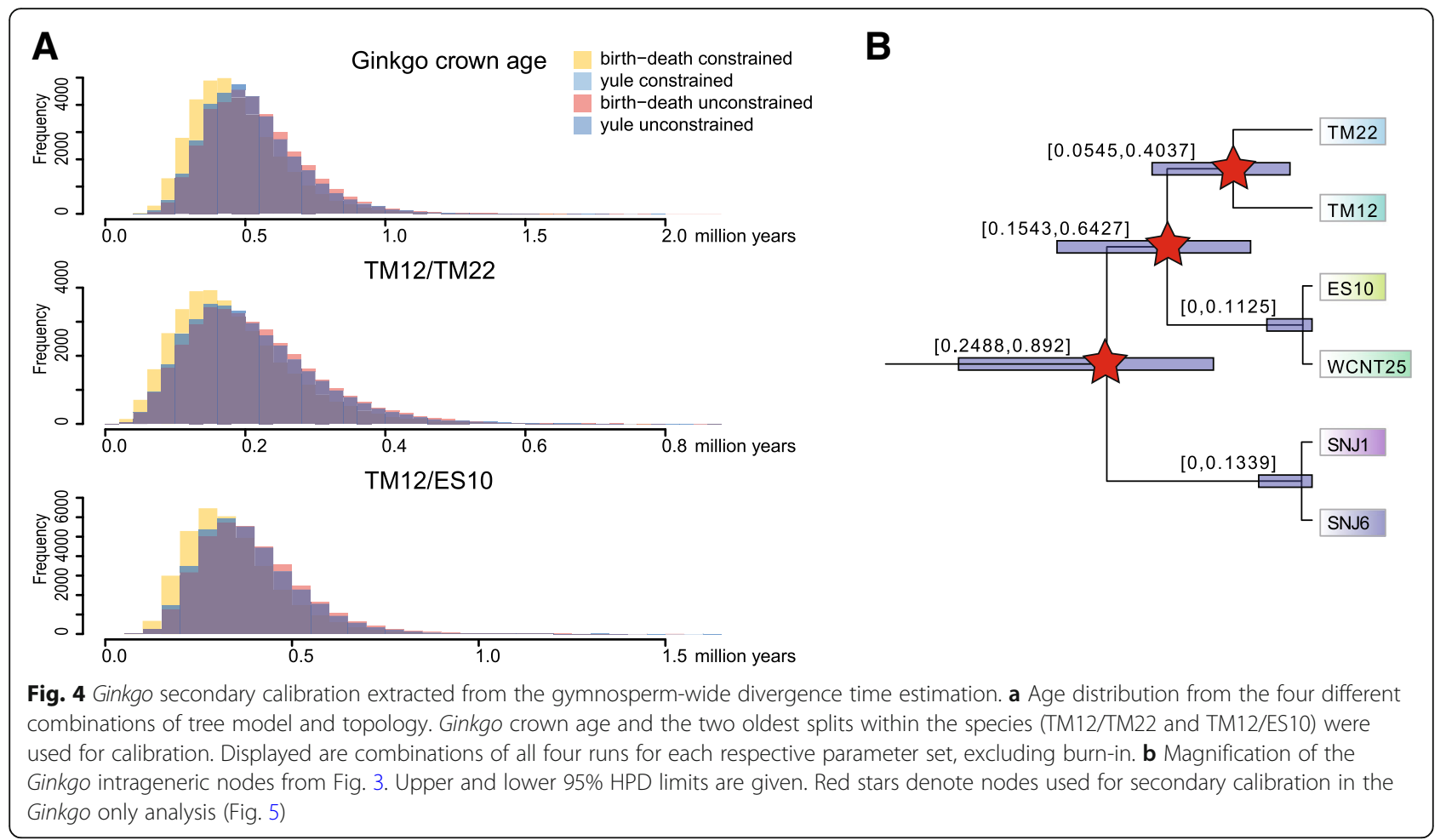

and gymnosperms) estimated within our study as 388 mya is in the same order of magnitude as demonstrated earlier with 360 mya [24] or 330 mya [43] or the coincidence with the ancestral seed plant Whole Genome Duplication (WGD छ) 320 mya [44]. A thorough and critical phylogenetic study focusing on divergence time estimates in cycads and testing branching process priors provided crown group estimates for cycad evolution between 274 and 280 mya [29] (240 mya in our study) and a Ginkgocycad split of 296-323 mya (324 mya in our study).

The crown group age of Ginkgo was estimated to 0.39 mya using the total Ginkgo SNP dataset (Fig. 6) and demonstrates remarkably that the deepest split among extant Ginkgo populations is dating back a few 100 thousands year only and ends abruptly with the third and fourth to last warming (Interglacial) phases (S3-S4, shortly interrupted by a cooling (glaciation) phase L4; Fig. 7; [45]). This indicates that global extinction and decline of Ginkgo all across the northern hemisphere (7-10 mya the fossil record disappeared from North America, end of Pliocene of about 2.5 mya from Europe) continued dramatically also in East Asia. Many fossils of Ginkgo from the Tertiary and Quaternary are from East Asia [1], however, the youngest Ginkgo fossils have been recorded from the late Pliocene and Pleistocene in Japan [1]. In China, there is no record of Ginkgo in sediments younger than Eocene. Therefore, it could well be that Ginkgo is just an immigrant from Japan to China between 2 and 0.39 mya. Based on limited sampling it seems very likely that old and extant trees in Japan and Korea were introduced from China by humans $[3,5]$. Consequently, the evolutionary history of Ginkgo biloba before the S3/S4 warming phase remains speculative.

In full agreement with our plastome phylogeny, DNA sequence variation of the entire rDNA locus is extremely low. The highly repetitive 35S ribosomal DNA (rDNA) sequences encoding $18 \mathrm{~S}-5.8 \mathrm{~S}-26 \mathrm{~S}$ ribosomal RNA very often show high levels of intragenomic uniformity despite frequent occurrence of multiple loci, paralogs and pseudogenes. The homogenization process is known as concerted evolution, but often non-concerted evolution has also been observed frequently associated with hybrid speciation and/or polyploidization [46, 47]. Ginkgo's sister group, the cycads, are characterized by an extraordinary diversity of rDNA repeats [48-50]. This is not only true for the entire cycads, but also for individual cycad species [50]. This feature has been explained by the large genome size (e.g., [51]), dense DNA methylation, frequent methylated cytosine deamination and multiple rDNA loci [50]. Furthermore, long retention times of divergent rDNA paralogs are hypothesized because of the long life-span of several hundred to more than one thousand years. However, Ginkgo biloba has a similarly large genome of $11.5 \mathrm{Gbp}[9,52]$, and it is also a longlived tree growing to similar ages. Furthermore, whole genome duplications (WGD) have been postulated for both lineages (Ginkgo and cycads) recently $[9,53]$, which can also contribute to rDNA loci duplications. It has 


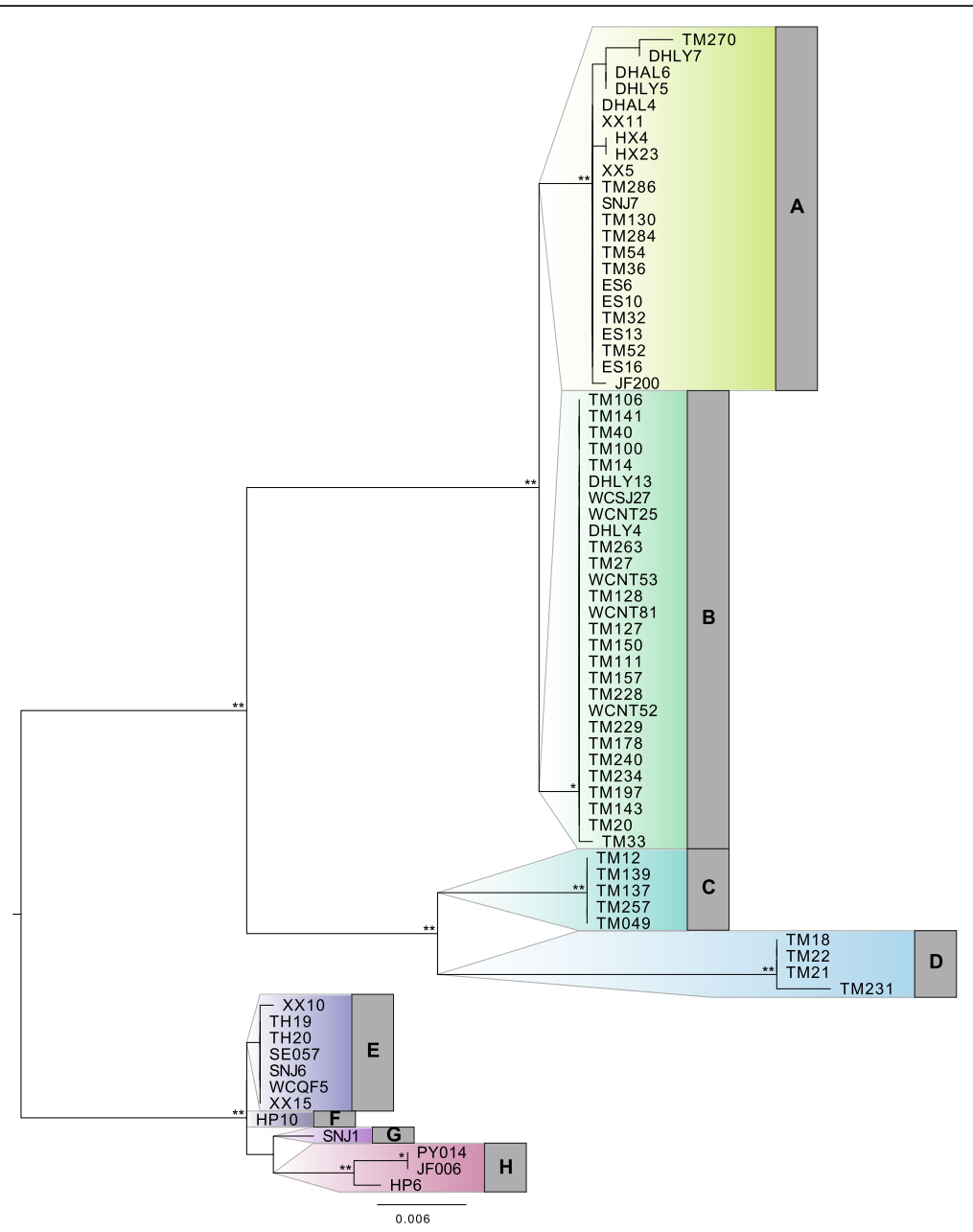

Fig. 5 Maximum likelihood phylogenetic reconstruction of 71 Ginkgo accessions. Bootstrap values from 1000 bootstrap replicates are indicated (** for bootstrap support of 100\% and * for support of 95-99\%). The clade containing SNJ1 and SNJ6 (i.e. haplotype groups E-H) was set as outgroup following the analysis in the gymnosperm-wide context

been demonstrated that this duplication most likely occurred about 300 mya [53], which correlates well with the stem group age of both lineages. This is, however, in sharp contrast to the finding that rDNA variation in Ginkgo biloba described for the entire locus analyzed and spanning 6448 bp was as low as in the annual selfer and angiosperm Arabidopsis thaliana [50]. Nucleotide diversity $(\pi)$ for intragenomic variation within the $18 \mathrm{~S}$ gene of Ginkgo biloba was calculated with less than $0.2 \%$ [50]; sequence ID: ERR845259/Illumina HiSeq2000, $170 \mathrm{bp}$ insert size, paired-end sequencing). Our results are fully congruent with this finding, and we did not even observe a higher rate when we compared between individuals considering the entire spatio-temporally structured gene pool. We may explain this at best with the high levels of outbreeding among a dioecious plant and a population structure in habitats largely maintaining genetic connectivity between individuals [16]. Hence, the results from our investigation of the rDNA region are in agreement with the conclusions based on plastid genomes and indicating (a) a very recent evolutionary history, and (b) repeated extensive gene flow and genetic coherence between refuge areas.

\section{Diversification of extant Ginkgo - Modes and tempi}

It is obvious that extant Ginkgo biloba must also reflect deep signatures of occurrence patterns of Chinese paleoendemics. In China 20 centers of plant endemism have been characterized [54]. In 35\% of these centers Ginkgo biloba is found and co-occurs with numerous other paleo-endemics (or even living fossils). Our data indicates that these centers date back several 100,000 years. During the last 400,000 years Ginkgo biloba underwent four cooling ("glaciation") cycles, herein named L4 to L1 [55]. For any of these cooling phases we observed respective phylogenetic splits. In contrast, no splits as indication for diversification were found during any of the warming phases S4 to S1 (Fig. 7). We interpret this 


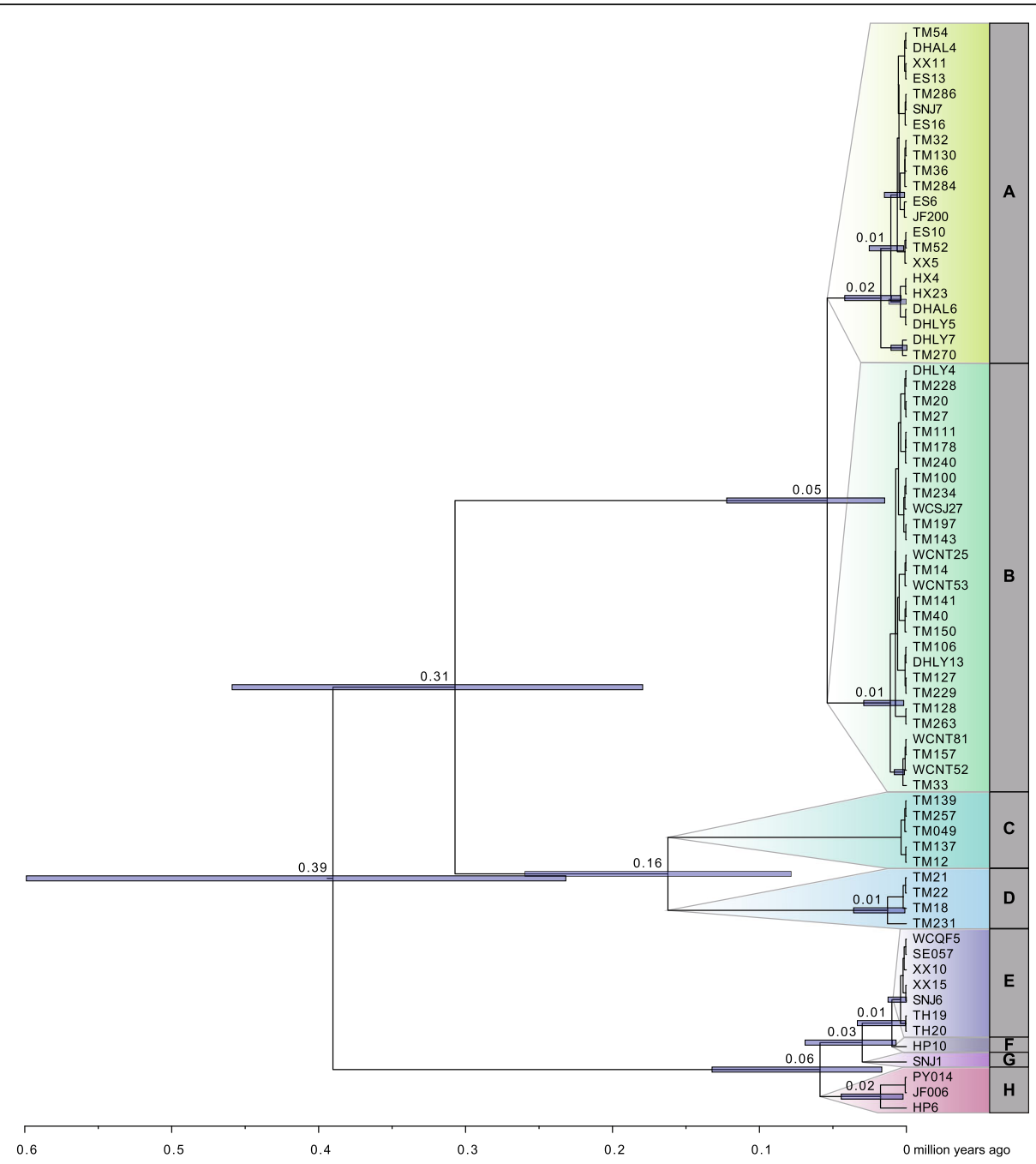

Fig. 6 Divergence time estimation of 71 Ginkgo accessions. Combined BEAST results from four independent MCMC runs of $1 * 10^{8}$ generations each. Calibration was based on secondary calibration derived from the gymnosperm-wide analysis. Divergence time estimates are shown with their 95\% HPD intervals for those nodes that represent splits between haplotypes in maximum likelihood reconstruction

pattern as a result of range expansion and increased population size during cooling phases. This increased the likelihood for new haplotype lineages to evolve and persist in a larger gene pool. With the onset of warming phases, the distribution range of Ginkgo biloba shrank, genetic drift increased and plastid haplotypes kept separated in refugia while small effective population sizes reduced the chance for new haplotypes to persist.

The temporally deep west-east dichotomous regional genetic structure that we observe in Ginkgo is apparent in numerous other temperate woody and herbaceous woodland plants from eastern Asia (Taxus wallichiana, Quercus variabilis, Kalopanax septemlobus, Schima superba, Bubleurum longiradiatum [12, 18, 19, 56]). Among all of these taxa, the ages of the divisions vary substantially, from 1.45 mya and 1.14 mya in Q. variabilis and B. longiradiatum, respectively, to $0.28-0.74$ mya and 0.61 mya in K. septemlobus and S. superba, respectively. These examples show that the same large-scale biogeographical pattern evolved several times during cooling and warming cycles and it is likely that presentday regional genetic variation integrates footprints of various cycles of past expansion and retraction. Reconstruction of these sequential processes might be further complicated by varying spatial patterns. In case of Eastern Asian forest biota major shifts towards the North and the South occurred during Pleistocene cooling (glaciation)/warming (deglaciation) cycles and may have also largely influenced temperate deciduous forest species. However, in the case of Ginkgo biloba this remains open. We hope that our work will stimulate future studies using genome-wide SNP data to elaborate in detail on population demographic history and processes, e.g. by estimating dynamics of effective population sizes along 


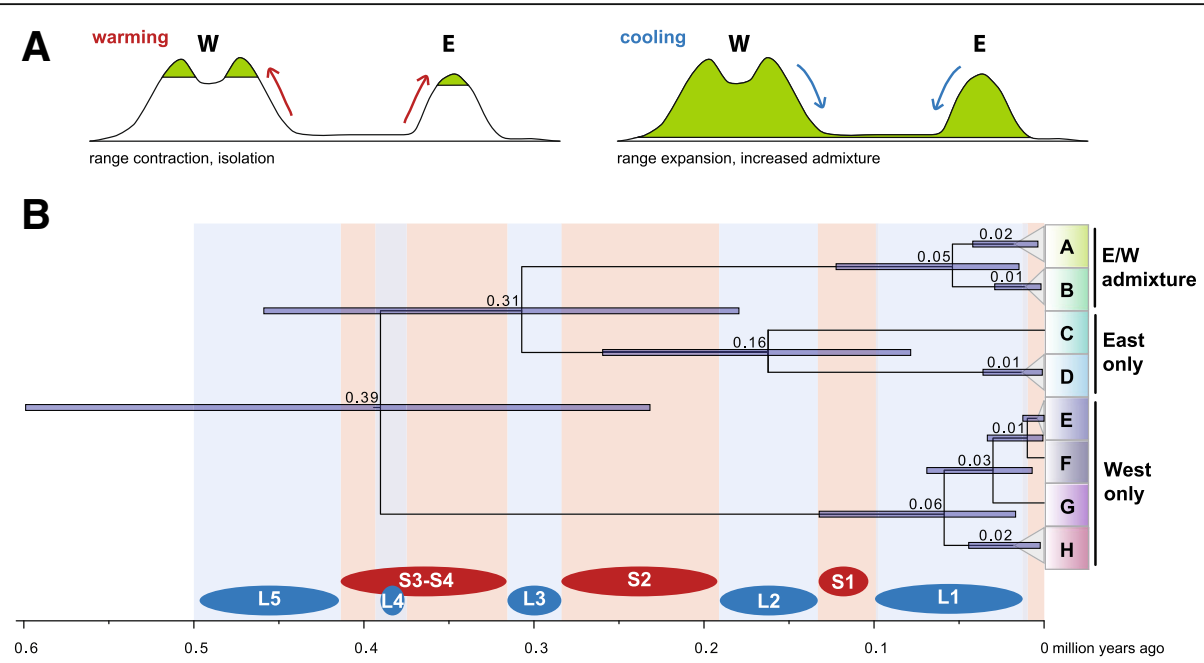

Fig. 7 Schematic timeline of Ginkgo evolution. a Graphical representation of our hypothesis of range contraction and isolation during warming phases and range expansion and admixture during cooling phases. b Simplification of divergence time estimations and branching patterns as shown in Fig. 6. Respective warming and cooling phases (S1-S4 and L1-L5 [55]) are indicated

the geological times of the last 400,000 years and correlating fluctuating effective population sizes with alternating cooling and warming phases while testing different coalescent models.

\section{Conclusion}

We shed some light on the early evolutionary history of Ginkgoatae and estimated a stem group age of approximately 325 mya. Analysis of whole plastid genome sequence data representing the entire spatio-temporal genetic variation of wild extant Ginkgo populations revealed the deepest temporal footprint of living populations dating back to approximately 390,000 years ago. Ginkgo biloba shows significant genetic structure among past refuge areas, and present-day directional West-East admixture of genetic diversity is shown to be the result of pronounced effects of the cooling period during the last glaciation approximately 100 to 20 kya. Ginkgo phylogenetic reconstruction and divergence time estimation revealed successive divergence synchronized with the Pleistocene cooling phases, and we hope that our evolutionary framework will serve as a conceptual roadmap for forthcoming genomic experiments providing deeper insights into the demographic history of contemporary Ginkgo.

\section{Methods}

\section{Plant material and sampling strategy}

We collected 71 samples of Ginkgo biloba from across its native distribution range in China [3,5]. With no extant close relatives of Ginkgo available, we used a dataset of complete chloroplast genomes available from Genbank/ENA, covering all major clades of gymnosperms, to set the evolution of this living fossil into context. Amborella trichopoda as a representative of the angiosperms was used as outgroup. In total, we included 43 published chloroplast genomes. For details see Additional files 1 and 4.

\section{DNA extraction and sequencing}

Total genomic DNA was extracted from silica-dried leaf material using the Invisorb Spin Plant Mini Kit (STRATEC Biomedical AG, Birkenfeld, Germany). Initial homogenization of the material was performed with 2 . $5 \mathrm{~mm}$ glass beads in a Precellys ${ }^{\circ} 24$ homogenizer (Bertin Technologies, Montigny-le-Brettonneux, France) in two intervals of $15 \mathrm{~s}$ at $5000 \mathrm{rpm}$. Hereafter we followed the extraction steps as given in the manufacturer's instructions including the optional RNAse digestion step.

DNA samples were checked for sufficient quality using gel electrophoresis and for concentration on a $\mathrm{Qubit}^{\circ} 2.0$

Table 1 Summary statistics of variable sites in the 35S rDNA operon. Intragenomic variables sites include all sites variable within at least one individual, intergenomic variable sites summarize sites with fixed differences (Additional file 3)

\begin{tabular}{|c|c|c|c|c|c|c|}
\hline & 185 rDNA & ITS1 & $5.8 \mathrm{~S} \mathrm{rDNA}$ & ITS2 & $25 \mathrm{~s}$ rDNA & total \\
\hline Sequence length (bp) & 1716 & 822 & 163 & 243 & 3411 & 6355 \\
\hline No. intragenomic variable sites & 3 & 6 & 2 & 4 & 21 & 36 \\
\hline No. intergenomic variable sites & & 1 & & 2 & 1 & 4 \\
\hline Variable ratio (\%) & 0.175 & 0.852 & 1.227 & 2.47 & 0.645 & 0.629 \\
\hline
\end{tabular}


fluorometer with the Qubit $^{\circ}$ dsDNA HS Assay Kit (Thermo Fisher Scientific, Waltham, Massachusetts, USA) before libraries were prepared and sequenced at the CellNetworks Deep Sequencing Core Facility (Heidelberg). 100 to $500 \mathrm{ng}$ of starting material were fragmented on a Covaris S2 Instrument. 63 libraries were prepared using the Ovation Ultra Low DR Multiplex kit 1-96 (NuGEN Technologies, Inc., San Carlos, California, U.S.), and two samples with the NEBnext Ultra DNA kit (New England Biolabs, Inc., Ipswich, Massachusetts, U. S.) with insert sizes ranging from 200 to $400 \mathrm{bp}$. The 63 and two library sets were then sequenced in $100 \mathrm{bp}$ paired-end mode on one lane of an Illumina HiSeq 2000 sequencing system (Illumina, Inc., San Diego, California, U.S.) each. Additionally, we included data of eight samples from Genbank/ENA study PRJNA307658. Raw sequencing reads generated for this study are available at Genbank/ENA under study PRJEB23626.

\section{Assembly and annotation of plastid genomes}

Illumina raw sequencing reads were filtered by trimming adapters and retaining sequence segments of 50 or more consecutive bases with quality scores 20 or higher to produce high-quality (HQ) sequences suitable for mapping and assembly. We assembled the complete plastid genome of each sample by using an initial mapping of HQ sequences to the Ginkgo biloba chloroplast sequence AB684440.1 [57], followed by iterative resolution steps combining automated consensus resolution, gap filling, dynamic remapping of all HQ sequences, interactive edition and assembly visualization. Sequence filtering, read mapping, plastome multi-alignments and annotation were performed with NUCLEAR version 3.2.4 (GYDLE Inc., Québec, Canada). Sequence assembly resolution, edition, visualization and polymorphism identification were done with VISION version 2.6.12 (GYDLE Inc., Québec, Canada). The mapping parameters used to recruit sequences into the plastid assembly (first from the common AB684440.1 reference, then from the iteratively-resolved assemblies) were: -140 -s 38 -m 1 -min-pct-cov 80 . This selected High-scoring Segment Pairs (HSP = gapless local alignment that achieves the requested alignment scores) of 40 bases or more, containing 38 consecutive identities and containing at most 1 mismatch every 40 bases (amounting to $97.5 \%$ local similarity) that were combined into alignments (combination of HSPs with possible gaps between them) covering at least $80 \%$ of the fragment's read sequence (on at least one side for paired reads). The resolving phases of the assembly used local realignments with parameters: -1 30 -s 16 -m 3 -min-score-cov 50 (HSP length > = 30, 16 consecutive identities, $90 \%$ local similarity, alignment covering 50 or more bases) to capture differences (fixed mutations and indels) within the alignments prior to resolving the assembly sequence. The assembler always keeps track of the mapping score of each read (in particular which mapped reads are perfectly aligned and which are not), therefore regions consistently connected and covered by strongly aligned reads are not subject to mis-assembly influenced by additional divergent reads, such as those representing insertions into the nuclear genome (which in addition to being divergent and unconnected to the main assembly have also much lower coverage).

The finishing step included the determination of exact junction sequences of inverted repeats and the assignment of a consistent starting position for all the plastomes. The average sequence coverage of plastome assemblies was $102 \times$ (minimum $9 \times$, maximum $845 \times$ ), with 58 out of 71 samples covered at $40 \times$ or more. Given the extreme similarity of plastome sequences among Ginkgo individuals, we performed two additional curation steps to eliminate sequence artifacts that could affect downstream phylogenetic studies. For this, we aligned all complete assemblies together and reviewed all polymorphisms in the context of their supporting aligned reads in the assembly. First, we identified 20 regions, comprising about 490 bases in all samples, where polymorphisms involved long A/T homopolymers with multiple individuals having inconclusive homopolymer length due to Illumina sequencing limitations. We discarded these regions for the network reconstruction of Ginkgo samples. Second, among the 34 mutations and indels specific to a single sample, 18 were documented by very low fragment coverage (often a single read) and were rejected due to possible sequencing error. To put the quality of our assemblies in context, the 18 possible artefacts identified in 71 samples can be compared with the sequence AB684440.1, used as our initial reference, which contains 12 indels and 4 single base mutations that are contradicted by all 71 samples, pointing to 16 sequence artefacts in that single sequence. Annotation of CDS, tRNAs and rRNAs in each sample was performed by extracting the nucleotide sequence of these features in the GenBank record of AB684440.1 and then aligning those to each complete plastome. Complete and annotated plastid genome sequences are available at GenBank/ ENA under accessions MG922594 to MG922643.

\section{General analytical strategy}

In order to estimate divergence times for our 71 Ginkgo samples, we employed a three-step process of analyses. 1) We reconstructed a network from the entire plastome sequence data of all 71 selected accessions spanning the entire distribution range in putative refuge areas and covering all previously defined haplotype groups and respective phylogenetic splits. 2) Based on the network, we selected a subset of accessions to be included in a gymnosperm-wide analysis using Amborella (angiosperms) as the outgroup. This analysis provided us with 
relationships within Ginkgo (for subsequent ingroup rooting) and secondary calibration points for Ginkgo biloba, because there is no fossil data available for calibrating the entire Ginkgoatae lineage. The deep divergence in this analysis spanning several hundred million years required us to focus on coding regions only. 3) Based on the results of (2), we expanded the analysis of (1) to phylogenetic reconstructions and BEAST divergence time estimation using the entire Ginkgo plastome dataset (with many more synapomorphic mutations) and including respective topological constraints and secondary divergence time calibration points. Among the various steps we cross-validated results comparing tree topologies and divergence times within our study using different models and among the various published studies.

\section{Step 1: Alignment and network reconstruction of Ginkgo samples}

All 71 Ginkgo plastid genomes were aligned with MAFFT v7.017 [58] as implemented in Geneious v7.1.7 (Biomatters Ltd., Auckland, New Zealand), using the FFT-NS-ix1000 algorithm (200 PAM/k=2 scoring matrix, gap open penalty $=1.53$, offset value $=0.123$ ) Subsequently, the alignment was partitioned into alignment blocks of single exons, introns and intergenic regions. Regions of low alignment quality were excluded using Gblocks v0.91b [59] using the following settings: no gaps were allowed, with minimum block length set to $1 \mathrm{bp}$. The second copy of the inverted repeat was excluded from further analyses, resulting in a total of 138,921 bp of sequence data. To account for rate heterogeneity among genes, the dataset was partitioned into subsets of genes evolving under the same evolutionary model and with similar substitution rates in PartitionFinder version 2.1.1 [60]. Bayesian information criterion (BIC) was used for model selection, and branch lengths between partitions were allowed to be unlinked. We tested only for partitioning by gene, and only for models implemented in BEAST. We found that our data was best run as a single partition with the GTR $+\Gamma+$ I model of evolution. A TCS network [61] was reconstructed using PopART (http://popart.otago.ac.nz). A haplotype map was drawn with packages 'maps' [62], 'mapdata' [63] and 'mapplots' [64] in $\mathrm{R}$ version 3.2.3 [65] using the 'worldHires' map. Consistency index and retention index were calculated in PAUP* version $4.0 \mathrm{~b} 10$ [26]. The complete alignment and PartitionFinder file as well as results are provided in Additional file 5.

\section{Step 2: Alignment, phylogenetic reconstruction and divergence time estimations of the gymnosperm-wide dataset}

To estimate Ginkgo crown age, we selected samples from six different haplotypes (indicated in Fig. 1) and combined them with gymnosperm plastid genome data. Because of high divergence between the included species only coding sequences were used for phylogenetic reconstruction and divergence time estimation of gymnosperms, and subsequently aligned in MAFFT with the same settings as above. Introns were excluded, as were start and stop codons in protein coding genes because of their tendency for higher levels of homoplasy. The final alignment blocks were realigned using MAFFT and manually inspected before they were subjected to an automated alignment quality control using Gblocks with the following settings: minimum number of sequences for conserved and flanking positions was set to 30 (60\%), allowed gap number was set to half, and minimum block length was set to 2 . Genes were excluded if they were not annotated in a majority of samples (e.g. rps16, psaM, $y c f 12$ ). The final dataset contained the coding regions of 78 genes (listed in Additional file 5), including four rRNAs, with a total alignment length of 60,520 bp. PartitionFinder was run to identify alignment blocks evolving under the same model and with a similar substitution rate. This resulted in five partitions, each of them evolving under the GTR $+\Gamma+$ I model. Alignment as well as PartitionFinder file and results are provided in Additional file 6. RAxML version 8.2.3 [27] was used to reconstruct a maximum likelihood (ML) phylogenetic tree. A rapid bootstrap analysis and subsequent ML search was conducted with 1000 bootstrap replicates. The partitioned dataset with five partitions as detected in PartitionFinder was used as input, with $G T R+\Gamma+I$ as nucleotide substitution model, and Amborella set as outgroup. The resulting phylogenetic reconstruction was displayed using FigTree version 1.4.1 [28].

BEAST version 1.7.5 [28] was used to estimate divergence times based on the five data partitions described above. As suggested by PartitionFinder, GTR $+\Gamma+\mathrm{I}$ was used as substitution model for all partitions with four gamma categories. The uncorrelated lognormal relaxed clock was used. We used the RAxML generated ML tree as starting tree after converting branch lengths to obtain a chronogram in $\mathrm{R}$ with package ape version 4.1 [66]. For fossil calibration we followed three recent studies on divergence times and divergence time estimation in land plants [43], in gymnosperms [25] and cycads in particular [29]. Fossil ages and their placement for calibration are listed in Additional file 7 and displayed in Additional file 8 . To constrain the root height of the tree we used secondary calibration, as no fossil was available for this node. By using a lognormal distribution with offset 249 mya, $\log ($ mean $) 4.4$ and $\log$ (stdev) 0.14 we matched the mean and 95\% HPD confidence intervals for the split of angiosperms and gymnosperms as estimated in [43]. We used uniform prior distributions for all fossil calibration points with maximum age 330 mya corresponding 
to the mean of the root height. Significant difference in estimated divergence times for cycads were reported when using different tree models [29], therefore we ran BEAST with both the yule [67] and the speciation: birthdeath process [68] model. Additionally, since BEAST resulted in a slightly different tree topology compared to the RAxML results, namely having Cycads and Ginkgo as sister to a clade of Pinaceae+(Gnetophytes+Cupressophytes) instead of Ginkgo being the sole sister group to this clade, we ran BEAST using two different tree topologies. Monophyly constraints were applied either only to all gymnosperms to set Amborella as outgroup (unconstrained datasets) or additionally to (Cupressaceae + Gnetales+Pinaceae+Ginkgo) to replicate the tree topology from RAxML with cycads as sister to all other gymnosperms. Altogether we analyzed 16 BEAST runs, as both the unconstrained and constrained dataset were run with both tree models four times each. MCMC chain length was 500 million generations, sampling parameters every 50,000 states, thus resulting in 2 billion generations per parameter set. Convergence of the chains as well as ESS values were evaluated in Tracer version 1.6.0 [28]. Output was combined in LogCombiner version 1.7 .5 [28], discarding the first $10 \%$ of every run as burn in, and subsequently annotated in TreeAnnotator version 1.7.5 [28] using median heights on a maximum clade credibility tree. FigTree was used to visualize the tree and 95\% HPD confidence intervals of node ages.

\section{Phylogenetic reconstruction and divergence time estimations within Ginkgo}

A maximum likelihood phylogenetic tree of all 71 Ginkgo samples was reconstructed with RAxML. Following the results from the gymnosperm-wide BEAST analysis and network reconstruction, the clade containing samples SNJ1 and SNJ6 (haplotypes E-H) was set as outgroup. We ran a rapid bootstrap analysis and subsequent ML search with 1000 bootstrap replicates, with substitution model GTR $+\Gamma+$ I for the complete alignment, and finally displayed the phylogenetic tree using FigTree.

Considering the not significantly different results from different tree models tree topologies of the gymnosperms phylogenetic tree (Fig. 4 and Additional file 2), and also following Condamine et al. [29], we chose the results from the speciation birth-death tree with unconstrained tree topology (i.e. Ginkgo as sister to Cycads) for secondary calibration of Ginkgo. Three nodes were selected for calibration: The Ginkgo crown age and the two oldest splits within the genus (TM12/TM22 and TM12/ES10). To best represent the posterior distributions of estimated ages at these nodes we extracted the age from each sampled tree (excluding burn-in) and fitted lognormal distributions using the package fitdistrplus [69] in $\mathrm{R}$.

Divergence time estimation was conducted in BEAST. Following the results from the gymnosperm wide analysis, all samples from haplotypes E, F, G and H were set as outgroup through monophyly constraints on this clade as well as the clade of haplotypes A, B, C, and D. As suggested by PartitionFinder we set GTR $+\Gamma+\mathrm{I}$ as substitution model, and we used the lognormal relaxed clock as tree model. Lognormal distributions were used for all secondary calibration points, with $\log ($ mean $)-0$. 673 and $\log$ (stdev) 0.323 for the tree root height, $\log (-$ mean) -1.644 and $\log$ (stdev) 0.458 for split TM12/TM22 and $\log ($ mean $) \quad-0.992$ and $\log$ (stdev) 0.348 for split TM12/ES10. Four independent MCMC chains were run for 100 million generations, sampling every 10,000 generations. Convergence of chains and ESS values were checked in Tracer, then the four runs were combined in LogCombiner, discarding the first $10 \%$ of each run as burn-in. The median heights were annotated onto the maximum clade credibility tree using TreeAnnotator, and finally this tree was visualized in FigTree.

\section{Definition of glacial and interglacial periods}

In this study we used the global chronostratigraphical correlation table to extract information from Pleistocene cooling and warming phases in China [70]). Here we followed the nomenclature from the Chinese Loess Sequence for the last $500 \mathrm{ky}$ (S0-S5: warming periods; L1L4: cooling periods). This record is more appropriate to define cooling and warming periods in East Asia/China and thereby characterizing shifts between woodland and steppe vegetation [55].

\section{Assembly and annotation of the nuclear 35 S rDNA region}

59 of the Illumina datasets generated for this study were subjected to de-novo assembly using CLC Genomic Workbench version 6.9 (Qiagen bioinformatics, Qiagen GmbH, Hilden, Germany), with default settings. The resulting contigs were indexed for BLAST using makekblastdb from BLAST 2.2.28+ [71, 72] and searched for similarity to a sequence containing a partial 35S-rDNASequence (partial 18S-rDNA, ITS1, 3.8S-rDNA, ITS2, partial 25S-rDNA, GenBank/ENA accession number EU643829). This resulted in 59 best-matching contiguous sequences containing partial or complete 35S-rDNA sequences, which were reverse-complemented where necessary using revseq in EMBOSS 6.6.0.0 [73]. By sequence similarity and quality checking in CLC Genomic Workbench a high quality complete sequence was chosen (sample TM14) and used as a reference. All samples' Illumina reads where then mapped to this reference sequence using bwa version 0.7.5a [74]. After quality 
filtering and deduplication with samtools version 1.6 [75], variants where called using freebayes version 1.1.0-50 [76] and consensus sequences extracted as FASTA with bcftools version 1.6 [75]. The resulting sequences where aligned using Clustal Omega [77], the alignment was checked and trimmed in PhyDE version 0.9971 [78]. Calculations were done using DNASp version 6.10.03 [79], VCFtools version 0.1.15 [80] and Microsoft Excel 2016.

\section{Additional files}

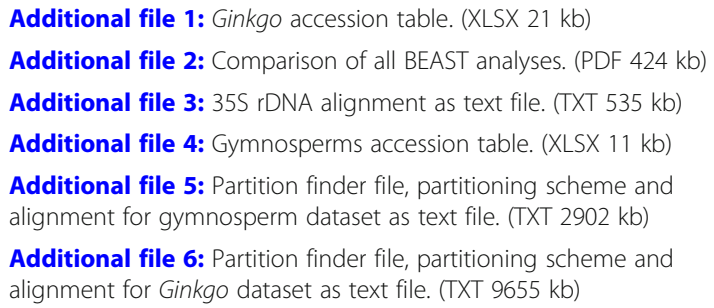

\section{Abbreviations}

Kya: Thousand years ago; ML: Maximum likelihood; Mya: Million years ago

\section{Acknowledgments}

We are grateful to David Ibberson as head of the Heidelberg University Campus Deep Sequencing Facility (Cellular Networks) for processing and sequencing libraries and providing respective raw data.

\section{Ethics and approval and consent to participate}

All plant material was collected in the wild, and no permissions are needed to obtain the material. Our study fully complies with regulations of CBD (convention biological diversity) and the Nagoya protocol. Detailed documentation of plant material is given by collection coordinates and collector with the accession list.

\section{Funding}

Funding from DAAD (German Academic Exchange Service) and CSC (Chinese Scholarship Council) under the PPP Germany-China program to MAK and CXF is appreciated. We acknowledge financial support by Deutsche Forschungsgemeinschaft (DFG) and Heidelberg University within the funding program Open Access Publishing and the National Key R\&D Program of China (2017YFA0605100). The funding body played no role in the design of the study and collection, analysis, and interpretation of data and in writing the manuscript.

\section{Availability of data and materials}

All datasets generated and/or analyzed during the current study are available at Genbank/ENA under studies PRJNA307658, PRJEB23626 and MG922594 to MG922643 as well as with Additional files 3, 5 and 6.

\section{Authors' contributions}

MAK designed the study and set up the experiments. NH, EW, PR, WZ, MK and MAK processed the data. PR processed the plastomes for submission to GenBank. NH, EW, PR, WZ, CXF, YPZ, MK and MAK analyzed and interpreted the data. YPZ, WZ and CXF contributed plant material. MAK and NH wrote the manuscript. All authors contributed to draft and have read and approved the final version of the manuscript.

\section{Competing interest}

The authors declare that they have no competing interests.

\section{Publisher's Note}

Springer Nature remains neutral with regard to jurisdictional claims in published maps and institutional affiliations.

\section{Author details}

${ }^{1}$ Center for Organismal Studies (COS) Heidelberg/Botanic Garden and Herbarium Heidelberg (HEID), University of Heidelberg, Im Neuenheimer Feld 345, D-69120 Heidelberg, Germany. ${ }^{2}$ Present address: Department of Environmental Sciences, Botany, University of Basel, Schönbeinstrasse 6, CH-4056 Basel, Switzerland. ${ }^{3}$ The Key Laboratory of Conservation Biology for Endangered Wildlife of the Ministry of Education, College of Life Sciences, Zhejiang University, Hangzhou 310058, China. ${ }^{4}$ GYDLE Inc., 1135 Grande Allée Ouest, Suite 220, QC, Québec G1S 1E7, Canada.

Received: 21 December 2017 Accepted: 13 April 2018

Published online: 27 April 2018

\section{References}

1. Uemura K. Cenozoic history of Ginkgo in East Asia. In: Hori T, Ridge RW, Tulecke W, Del Tredici P, Trémouillaux-Guiller J, Tobe H, editors. Ginkgo Biloba Glob Treasure Biol Med. Tokyo: Springer Japan; 1997. p. 207-21. Available from: https://doi.org/10.1007/978-4-431-68416-9_16.

2. Zhou Z-Y. An overview of fossil Ginkgoales. Palaeoworld. 2009;18:1-22.

3. Gong W, Chen C, Dobeš C, Fu C-X, Koch MA. Phylogeography of a living fossil: Pleistocene glaciations forced Ginkgo biloba L. (Ginkgoaceae) into two refuge areas in China with limited subsequent postglacial expansion. Mol Phylogenet Evol. 2008:48:1094-105.

4. Zhou Z, Zheng S. The missing link in Ginkgo evolution. Nature. 2003;423:821.

5. Zhao Y, Paule J, Fu C, Koch MA. Out of China: distribution history of Ginkgo biloba L. Taxon. 2010:59:495-504.

6. He S-A, Gu Y, Pang Z-J. Resources and prospects of Ginkgo biloba in China. Ginkgo biloba - A Global Treasure. Springer; 1997. p. 373-383.

7. Hori S, Hori T. A cultural history of Ginkgo biloba in Japan and the generic name ginkgo. Ginkgo biloba - A Global Treasure. Springer; 1997. p. 385-411.

8. Tsumura $Y$, Ohba $K$. The genetic diversity of isozymes and the possible dissemination of Ginkgo biloba in ancient times in Japan. Ginkgo biloba - A Global Treasure. Springer; 1997. p. 159-172.

9. Guan R, Zhao Y, Zhang H, Fan G, Liu X, Zhou W, et al. Draft genome of the living fossil Ginkgo biloba. GigaScience. 2016;5:49.

10. Qi X-S, Chen C, Comes HP, Sakaguchi S, Liu Y-H, Tanaka N, et al. Molecular data and ecological niche modelling reveal a highly dynamic evolutionary history of the east Asian tertiary relict Cercidiphyllum (Cercidiphyllaceae). New Phytol. 2012;196:617-30.

11. Guan B-C, Fu C-X, Qiu Y-X, Zhou S-L, Comes HP. Genetic structure and breeding system of a rare understory herb, Dysosma versipellis (Berberidaceae), from temperate deciduous forests in China. Am J Bot. 2010:97:111-22.

12. Gao LM, Möller M, Zhang X-M, Hollingsworth ML, LIU J, Mill RR, et al. High variation and strong phylogeographic pattern among cpDNA haplotypes in Taxus wallichiana (Taxaceae) in China and North Vietnam. Mol Ecol. 2007;16: 4684-98.

13. Qian H, Ricklefs RE. Large-scale processes and the Asian bias in species diversity of temperate plants. Nature. 2000;407:180.

14. Harrison SP, Yu G, Takahara H, Prentice IC. Diversity of temperate plants in East Asia. Nature. 2001;413:129.

15. Qiu Y-X, Fu C-X, Comes HP. Plant molecular phylogeography in China and adjacent regions: tracing the genetic imprints of quaternary climate and environmental change in the world's most diverse temperate flora. Mol Phylogenet Evol. 2011;59:225-44.

16. Zhao Y, Yan X, Muir G, Dai Q, Koch MA, Fu C. Incongruent range dynamics between co-occurring Asian temperate tree species facilitated by life history traits. Ecol Evol. 2016;6:2346-58.

17. Hamrick JL, Godt M. Effects of life history traits on genetic diversity in plant species. Philos Trans R Soc Lond Ser B Biol Sci. 1996;351:1291-8.

18. Zhao C, Wang C-B, Ma X-G, Liang Q-L, He X-J. Phylogeographic analysis of a temperate-deciduous forest restricted plant (Bupleurum longiradiatum Turcz.) reveals two refuge areas in China with subsequent refugial isolation promoting speciation. Mol Phylogenet Evol. 2013;68:628-43.

19. Chen D, Zhang X, Kang H, Sun X, Yin S, Du H, et al. Phylogeography of Quercus variabilis based on chloroplast DNA sequence in East Asia: multiple glacial refugia and mainland-migrated island populations. PLoS One. 2012;7: e47268. 
20. Feng L, Zhang Y-P, Chen X-D, Yang J, Zhou T, Bai G-Q, et al. Allopatric divergence, local adaptation, and multiple quaternary refugia in a long-lived tree (Quercus spinosa) from subtropical China. bioRxiv. 2017;112375.

21. Gong W, Liu W, Gu L, Kaneko S, Koch MA, Zhang D. From glacial refugia to wide distribution range: demographic expansion of Loropetalum chinense (Hamamelidaceae) in Chinese subtropical evergreen broadleaved forest. Org Divers Evol. 2016;16:23-38.

22. Qin A, Liu B, Guo Q, Bussmann RW, Ma F, Jian Z, et al. Maxent modeling for predicting impacts of climate change on the potential distribution of Thuja sutchuenensis Franch., an extremely endangered conifer from southwestern China. Glob Ecol Conserv. 2017;10:139-46.

23. Fan $D$, Hu W, Li B, Morris AB, Zheng M, Soltis DE, et al. Idiosyncratic responses of evergreen broad-leaved forest constituents in China to the late quaternary climate changes. Sci Rep. 2016;6:31044.

24. Clarke JT, Warnock RCM, Donoghue PCJ. Establishing a time-scale for plant evolution. New Phytol. 2011;192:266-301.

25. Lu Y, Ran J-H, Guo D-M, Yang Z-Y, Wang X-Q. Phylogeny and divergence times of gymnosperms inferred from single-copy nuclear genes. PLoS One. 2014:9:e107679. Buerki S, editor

26. Swofford D. PAUP*: phylogenetic analysis using parsimony $\left({ }^{*}\right.$ and other methods). Sunderland: Sinauer Associates; 2002.

27. Stamatakis A. RAxML version 8: a tool for phylogenetic analysis and postanalysis of large phylogenies. Bioinformatics. 2014;30:1312-3.

28. Drummond AJ, Suchard MA, Xie D, Rambaut A. Bayesian Phylogenetics with BEAUti and the BEAST 1.7. Mol Biol Evol. 2012;29:1969-73.

29. Condamine FL, Nagalingum NS, Marshall CR, Morlon H. Origin and diversification of living cycads: a cautionary tale on the impact of the branching process prior in Bayesian molecular dating. BMC Evol Biol. 2015;15:65.

30. Mathews S. Phylogenetic relationships among seed plants: persistent questions and the limits of molecular data. Am J Bot. 2009:96:228-36.

31. Mathews S, Clements MD, Beilstein MA. A duplicate gene rooting of seed plants and the phylogenetic position of flowering plants. Philos Trans R Soc B Biol Sci. 2010;365:383.

32. Li Z, De La Torre AR, Sterck L, Cánovas FM, Avila C, Merino I, et al. Singlecopy genes as molecular markers for phylogenomic studies in seed plants. Genome Biol Evol. 2017;9:1130-47.

33. Xi Z, Rest JS, Davis CC. Phylogenomics and coalescent analyses resolve extant seed plant relationships. PLoS One. 2013:8:e80870.

34. Wu C-S, Chaw S-M, Huang Y-Y. Chloroplast phylogenomics indicates that Ginkgo biloba is sister to cycads. Genome Biol Evol. 2013;5:243-54.

35. Burleigh JG, Mathews S. Phylogenetic signal in nucleotide data from seed plants: implications for resolving the seed plant tree of life. Am J Bot. 2004;91:1599-613.

36. Wu C-S, Wang Y-N, Hsu C-Y, Lin C-P, Chaw S-M. Loss of different inverted repeat copies from the chloroplast genomes of Pinaceae and Cupressophytes and influence of heterotachy on the evaluation of gymnosperm phylogeny. Genome Biol Evol. 2011;3:1284-95.

37. Zhong B, Deusch O, Goremykin W, Penny D, Biggs PJ, Atherton RA, et al. Systematic error in seed plant phylogenomics. Genome Biol Evol. 2011;3:1340-8.

38. Ran J-H, Gao H, Wang X-Q. Fast evolution of the retroprocessed mitochondrial rps3 gene in conifer II and further evidence for the phylogeny of gymnosperms. Mol Phylogenet Evol. 2010;54:136-49.

39. Cibrián-Jaramillo A, De la Torre-Bárcena JE, Lee EK, Katari MS, Little DP, Stevenson DW, et al. Using phylogenomic patterns and gene ontology to identify proteins of importance in plant evolution. Genome Biol Evol. 2010;2:225-39.

40. Wickett NJ, Mirarab S, Nguyen N, Warnow T, Carpenter E, Matasci N, et al. Phylotranscriptomic analysis of the origin and early diversification of land plants. Proc Natl Acad Sci. 2014;111:E4859-68.

41. One Thousand Plant Transcriptomes Initiative. A phylogenetic view of evolutionary complexity in Green Plants. Nature (under review)

42. Li Z, Baniaga AE, Sessa EB, Scascitelli M, Graham SW, Rieseberg LH, et al. Early genome duplications in conifers and other seed plants. Sci Adv. 2015; 1:e1501084. Available from: http://advances.sciencemag.org/content/1/10/ e1501084.abstract

43. Magallón S, Hilu KW, Quandt D. Land plant evolutionary timeline: gene effects are secondary to fossil constraints in relaxed clock estimation of age and substitution rates. Am J Bot. 2013:100:556-73.

44. Jiao Y, Wickett NJ, Ayyampalayam S, Chanderbali AS, Landherr L, Ralph PE, et al. Ancestral polyploidy in seed plants and angiosperms. Nature. 2011; 473:97.

45. Gibbard P. Cohen KM. Global chronostratigraphical correlation table for the last 2.7 million years. Episodes. 2008;31:243-7.
46. Koch MA, Dobeš C, Mitchell-Olds T. Multiple hybrid formation in natural populations: concerted evolution of the internal transcribed spacer of nuclear ribosomal DNA (ITS) in north American Arabis divaricarpa (Brassicaceae). Mol Biol Evol. 2003;20

47. Harpke D, Peterson A. Non-concerted ITS evolution in Mammillaria (Cactaceae). Mol Phylogenet Evol. 2006:41:579-93.

48. Xiao L-Q, Möller M. Nuclear ribosomal its functional paralogs resolve the phylogenetic relationships of a late-Miocene radiation cycad Cycas (Cycadaceae). PLoS One. 2015;10:e0117971.

49. Xiao L-Q, Möller M, Zhu H. High nrDNA ITS polymorphism in the ancient extant seed plant Cycas: incomplete concerted evolution and the origin of pseudogenes. Mol Phylogenet Evol. 2010;55:168-77.

50. Wang W, Ma L, Becher H, Garcia S, Kovarikova A, Leitch IJ, et al. Astonishing 355 rDNA diversity in the gymnosperm species Cycas revoluta Thunb. Chromosoma. 2016:125:683-99.

51. Leitch A, Leitch I. Ecological and genetic factors linked to contrasting genome dynamics in seed plants. New Phytol. 2012;194:629-46.

52. Bennett M, Leitch I. Angiosperm DNA C-values database (release 8.0, Dec. 2012). Website http://data.kew.org/cvalues. 2012

53. Roodt D, Lohaus R, Sterck L, Swanepoel RL, Van de Peer Y, Mizrachi E. Evidence for an ancient whole genome duplication in the cycad lineage. PLoS One. 2017;12:e0184454

54. López-Pujol J, Zhang F-M, Sun H-Q, Ying T-S, Ge S. Centres of plant endemism in China: places for survival or for speciation? J Biogeogr. 2011; 38:1267-80.

55. Maher BA. Palaeoclimatic records of the loess/palaeosol sequences of the Chinese loess plateau. Quat Sci Rev. 2016;154:23-84.

56. Sakaguchi S, Qiu YX, Liu YH, Qi XS, Kim SH, Han J, et al. Climate oscillation during the quaternary associated with landscape heterogeneity promoted allopatric lineage divergence of a temperate tree Kalopanax septemlobus (Araliaceae) in East Asia. Mol Ecol. 2012;21:3823-38.

57. Lin C-P, Wu C-S, Huang Y-Y, Chaw S-M. The complete chloroplast genome of Ginkgo biloba reveals the mechanism of inverted repeat contraction. Genome Biol Evol. 2012:4:374-81.

58. Katoh K, Standley DM. MAFFT multiple sequence alignment software version 7: improvements in performance and usability. Mol Biol Evol. 2013; 30:772-80.

59. Castresana J. Selection of conserved blocks from multiple alignments for their use in phylogenetic analysis. Mol Biol Evol. 2000;17:540-52.

60. Lanfear R, Calcott B, Kainer D, Mayer C, Stamatakis A. Selecting optimal partitioning schemes for phylogenomic datasets. BMC Evol Biol. 2014;14:82.

61. Clement M, Posada D, Crandall KA. TCS: a computer program to estimate gene genealogies. Mol Ecol. 2000;9:1657.

62. Becker OS code by RA, Minka ARWR version by RBE by TP, Deckmyn A. maps: Draw Geographical Maps. 2017. Available from: https://CRAN.Rproject.org $/$ package $=$ maps

63. Becker OS code by RA, Brownrigg ARWR version by R. mapdata: extra map databases. 2016. Available from: https://CRAN.R-project.org/package=mapdata

64. Gerritsen H. mapplots: data visualisation on maps. 2014. Available from: https://CRAN.R-project.org/package=mapplots

65. R Core Team. R: a language and environment for statistical computing. Vienna: R Foundation for Statistical Computing; 2016. Available from: https://www.R-project.org/

66. Paradis E, Claude J, Strimmer K. APE: analyses of Phylogenetics and evolution in R language. Bioinformatics. 2004;20:289-90.

67. Gernhard T, Hartmann K, Steel M. Stochastic properties of generalised yule models, with biodiversity applications. J Math Biol. 2008;57:713-35.

68. Stadler T. Sampling-through-time in birth-death trees. J Theor Biol. 2010; 267:396-404.

69. Delignette-Muller ML, Dutang C. Fitdistrplus: an R package for fitting distributions. J Stat Softw. 2015;64:1-34.

70. Cohen KM, Gibbard PM. Global chronostratigraphical correlation table for the last 2.7 million years, v. 2016a. 2016. Cambridge: Subcommission on quaternary Stratigraphy, International Commission on Stratigraphy; 2016. Available from: http://www.stratigraphy.org/index.php/ics-chart-timescale

71. Altschul SF, Gish W, Miller W, Myers EW, Lipman DJ. Basic local alignment search tool. J Mol Biol. 1990;215:403-10.

72. Camacho C, Coulouris G, Avagyan V, Ma N, Papadopoulos J, Bealer K, et al. BLAST+: architecture and applications. BMC Bioinformatics. 2009;10:421.

73. Rice P, Longden I, Bleasby A. EMBOSS: the European molecular biology open software suite. Elsevier current trends; 2000. 
74. Li H, Durbin R. Fast and accurate short read alignment with burrowswheeler transform. Bioinformatics. 2009;25:1754-60.

75. Li H, Handsaker B, Wysoker A, Fennell T, Ruan J, Homer N, et al. The sequence alignment/map format and SAMtools. Bioinformatics. 2009;25: 2078-9.

76. Garrison E, Marth G. Haplotype-based variant detection from short-read sequencing. ArXiv Prepr ArXiv12073907. 2012;

77. Sievers F, Wilm A, Dineen D, Gibson TJ, Karplus K, Li W, et al. Fast, scalable generation of high-quality protein multiple sequence alignments using Clustal omega. Mol Syst Biol. 2011;7:539.

78. Müller J, Müller K, Neinhuis C, Quandt D. PhyDE-Phylogenetic Data Editor. Program Distrib Authors Httpbackslashbackslashwww Phyde De. 2005;

79. Rozas J, Ferrer-Mata A, Sánchez-DelBarrio JC, Guirao-Rico S, Librado P, Ramos-Onsins SE, et al. DnaSP 6: DNA sequence polymorphism analysis of large data sets. Mol Biol Evol. 2017;34:3299-302.

80. Danecek P, Auton A, Abecasis G, Albers CA, Banks E, DePristo MA, et al. The variant call format and VCFtools. Bioinformatics. 2011;27:2156-8.

Ready to submit your research? Choose BMC and benefit from:

- fast, convenient online submission

- thorough peer review by experienced researchers in your field

- rapid publication on acceptance

- support for research data, including large and complex data types

- gold Open Access which fosters wider collaboration and increased citations

- maximum visibility for your research: over $100 \mathrm{M}$ website views per year

At BMC, research is always in progress.

Learn more biomedcentral.com/submissions 